\title{
Indicadores de ciência, tecnologia e inovação: mensuração dos sistemas de CTeI nos estados brasileiros*
}

\author{
Elisa Maria Pinto Rocha \\ Economista, doutora em ciência da informação pela Universidade \\ Federal de Minas Gerais; pesquisadora e professora do Programa de \\ Mestrado em Administração Pública da Fundação João Pinheiro. \\ E-mail: elisa.lirocha@fjp.mg.gov.br \\ Marta Araújo Tavares Ferreira \\ Professora do Programa de Pós-graduação em Ciência da Informação \\ da Universidade Federal de Minas Gerais (PPGCI-UFMG). \\ E-mail: maraujo@ufmg.br
}

\begin{abstract}
Resumo
Este artigo trata da construção de uma medida que possibilite caracterizar e classificar os sistemas de inovação existentes nos estados brasileiros. A partir de bases de dados e informações já existentes, elabora-se o Índice de Ciência, Tecnologia e Inovação para os estados das regiões Sudeste e Sul do país. Os resultados obtidos sugerem a existência de pelo menos quatro "estágios" de desenvolvimento dos sistemas estaduais de inovação na região. No "estágio" mais avançado estaria posicionado São Paulo; Rio de Janeiro, Rio Grande do Sul e Santa Catarina estariam na posição imediatamente abaixo de São Paulo; Paraná e Minas Gerais ocupariam posições menos confortáveis, enquanto o Espirito Santo corresponderia a um estágio menos avançado.
\end{abstract}

\section{Palavras-chave}

Ciência e tecnologia; Indicadores de inovação; Minas Gerais.

\section{Indicators of science, technology and innovation : measurement of STel systems in the Brazilian States}

\begin{abstract}
This article discusses the building up of measurement patterns that will allow characterization and classification of innovation systems found in Brasizilian States. From database information currently available in the country, Science, Technology and Innovation Index is obtained for the South and Southeast Regions of the Country. The results suggest the existence of at least four "stages" in the development of the innovation state systems in the region. São Paulo is in the most advanced "stage"; Rio de Janeiro, Rio Grande do Sul and Santa Catarina come right after São Paulo; Paraná and Minas Gerais are in less comfortable positions, whereas Espírito Santo is in the least advanced "stage".
\end{abstract}

\section{Keywords}

Science and technology; Innovation indicators; Minas Gerais.

\footnotetext{
* Artigo baseado nos resultados da tese de doutoramento defendida junto ao Programa de Pós-graduação em Ciência da Informação da Universidade Federal de Minas Gerais (PPGCI), em outubro de 2003.
}

\section{INTRODUÇÃO}

No atual momento vivenciado pela sociedade contemporânea, há um reconhecimento de que a ciência, tecnologia e inovação constituem-se fatores diferenciadores do desenvolvimento social e econômico de países e regiões.

O Brasil e a maioria dos países latino-americanos dispõem de indicadores que possibilitam maior conhecimento sobre a posição relativa dos estados e municípios no plano econômico (a exemplo do Produto Interno Bruto Per Capita, PIB), bem como no plano geral do desenvolvimento humano (a exemplo do Desenvolvimento Humano, IDH).

Entretanto, na esfera da ciência e tecnologia, o país ainda não possui um instrumento de informação capaz de caracterizar e dimensionar os sistemas estaduais de ciência, tecnologia e inovação.

Um dos objetivos desta pesquisa é propor a construção de um índice desta natureza, a partir da utilização de bases de dados e informações já existentes na área de ciência e tecnologia, compiladas por instituições como ministérios, agências governamentais e associações privadas, tais como Ministério da Ciência e Tecnologia (MCT), Conselho Nacional de Desenvolvimento Científico e Tecnológico (CNPq), Associação Nacional de Pesquisa, Desenvolvimento e Engenharia das Empresas Inovadoras (Anpei) e Associação Nacional de Entidades Promotoras de Empreendimentos de Tecnologias Avançadas (Anprotec), dentre outras.

Indicadores como o PIB Per Capita e o IDH indicam que Minas Gerais, o terceiro maior estado da federação no plano econômico, apresenta, entretanto, posição pouco confortável em termos de desenvolvimento econômico e de desenvolvimento humano: dentre as 27 unidades da federação, é o $11^{\circ}$ estado no que se refere ao PIB Per Capita e ao IDH. Comparando-se os estados integrantes das grandes regiões brasileiras, Minas Gerais detém a mais desfavorável posição dentre os estados do Sudeste-Sul do país no que se refere a estes dois indicadores.

No que se refere ao plano da ciência, tecnologia e inovação, que posição ocupa Minas Gerais? Que fragilidades e 


\section{Elisa Maria Pinto Rocha / Marta Araújo Tavares Ferreira}

oportunidades apresenta o sistema de inovação do estado em relação a estados brasileiros?

Essas são questões que norteiam a nossa pesquisa e cujas respostas envolvem a construção e a análise do "índice de ciência, tecnologia e inovação" para os estados brasileiros que integram as regiões Sudeste e Sul (ICTeI). Além ampliar a compreensão sobre os sistemas de inovação regionais e locais, esta pesquisa representa um passo adicional aos esforços que ora estão sendo empreendidos por pesquisadores brasileiros e latino-americanos, no sentido de melhor entender as particularidades que a inovação tecnológica assume em espaços caracterizados por sistemas de inovação imaturos e incompletos, como é o caso brasileiro (Lastres e Albagli, 1999).

A experiência mundial no que se refere à construção de indicadores de ciência, tecnologia e inovação mostra que essa tarefa não se limita a apenas uma disciplina da área de conhecimento, como a economia ou a sociologia. Para além dessas, é fundamental integrar a visão e a experiência dos profissionais da informação retratadas pela bibliometria, ciênciometria e informetria, conforme se apreende, dentre outros, de Macias-Chapula (1998), Spinak (1998), Rousseau (1998), Vanti (2002) e Wormell (1998).

Ao envolver atividades como a identificação, consulta, busca, compilação e interpretação de dados e informações de fontes secundárias, produzidas por departamentos de estatística e equipes de profissionais que lidam com a produção de indicadores de CeT no Brasil, o nosso exercício de construção do ICTeI possibilita, também, maior conhecimento sobre as bases de dados de informação tecnológica atualmente disponíveis em instituições brasileiras*.

\section{METODOLOGIA}

A concepção geral que norteia a construção do ICTeI para os estados brasileiros se baseia na perspectiva dos "sistemas nacionais de inovação" que pode ser compreendido como uma construção institucional, produto seja de uma ação planejada e consciente, seja de um somatório de

\footnotetext{
"Dentre estas instituições, destacam-se como fontes de informação utilizadas na construção do ICTeI: Ministério da Ciência e Tecnologia (MCT), Conselho Nacional de Desenvolvimento Científico e Tecnológico (CNPq), Ministério da Educação (MEC), Ministério do Desenvolvimento Indústria e Comércio Exterior (MDIC), Instituto Brasileiro de Geografia e Estatística (IBGE), Fundação Sistema Estadual de Análise de Dados do Estado de São Paulo (Seade), Centro de Estatística e Informações da Fundação João Pinheiro (CEI/FJP) e Associação Nacional de Entidades Promotoras de Empreendimentos de Tecnologias Avançadas (Anprotec). Para maior discussão sobre informação tecnológica e uso de bases de dados de informação para negócios, ver Montalli e Campelo (1997), Souza e Borges (1996) e Cendón (2002), citados na bibliografia.
}

decisões não planejadas e desarticuladas, que impulsiona o progresso tecnológico (Freeman e Soete, 1997; Nelson, 1993).

A referência metodológica para a elaboração do ICTeI é o Technology Achievement Index (TAI), elaborado pelo United Nations Development Programme (UNDP ou PNUD, sigla em português) e divulgado no Human Development Report-2001**.

O relatório do desenvolvimento humano do PNUD - 2001 apresentou, pela primeira vez na história da instituição, a metodologia e o ranking mundial relativo ao Technology Achievement Index. O TAI (traduzido no Brasil como "índice de realização tecnológica") calculado para 72 países é um índice composto, que parte da consideração de três dimensões de grande relevância para os objetivos da política científica e tecnológica de um país: criação de tecnologia, difusão de inovações e habilidades humanas.

Estudos como aqueles realizados por Albuquerque (2001), Diniz e Lemos (1999) e Aguiar e Gonçalves (2000) e que discutem o sistema de inovação de Minas Gerais também se constituem referências importantes para a construção do ICTeI.

No caso do ICTeI, que nos propomos a elaborar, é importante ressaltar que, em função da inexistência ou carência de dados passíveis de comparação entre os estados brasileiros, houve necessidade de adaptações à metodologia de construção do índice desenvolvido pelo PNUD. Nesse sentido, o ICTeI é metodologicamente diferente do TAI e pode ser compreendido como uma medida indireta dos sistemas estaduais de inovação, obtida a partir de um conjunto de indicadores que retrata as dimensões mais fundamentais deste sistema. O ICTeI é, simultaneamente, um produto e um insumo de informação que fornece parâmetros para a caracterização e comparação dos sistemas de inovação estaduais.

\section{Construção do ICTeI Para os estados do Sudeste-Sul}

O índice por nós desenvolvido focaliza quatro dimensões principais de um sistema de inovação estadual: prioridade governamental à área de ciência e tecnologia; produção científica e tecnológica; base educacional e disponibilidade de recursos humanos qualificados; amplitude e difusão da inovação no âmbito das empresas localizadas no estado.

\footnotetext{
** O detalhamento da metodologia de construção do TAI e a discussão sobre os resultados do índice encontram-se em United Nations Development Programme (UNDP), citado na bibliografia.
} 
O significado dessas dimensões, os indicadores selecionados para representá-las - ou seja, os indicadores utilizados como substitutos - e as fontes de informação utilizadas na sua elaboração são descritos a seguir.

\section{A) Prioridade governamental À CiÊnCia e teCnologia}

O investimento público no campo científico e tecnológico é um vetor fundamental do desenvolvimento socioeconômico de países e regiões e constitui um dos principais condicionantes da competitividade empresarial. Em países caracterizados por sistemas nacionais de inovação imaturos, como é o caso do Brasil, os gastos realizados pelo poder público para o desenvolvimento científico e tecnológico assumem relevância ainda maior, devido aos baixos dispêndios efetuados pelas empresas privadas.

Nesta pesquisa, os seguintes indicadores foram utilizados como proxies dessa dimensão:

- Gasto Per Capita Governamental em Ciência e Tecnologia: corresponde à soma do dispêndio federal efetuado no estado, pelo Conselho Nacional de Desenvolvimento Científico e Tecnológico (CNPq) e do gasto realizado pelo governo estadual com a função ciência e tecnologia, divido pela população do estado (R\$ por habitante).

Os dados sobre gastos federais do CNPq nos estados (para apoio e fomento da pesquisa científica) referem-se ao ano 2000 e foram obtidos na página do órgão/seção Resenha Estatística 1995-2000, acessada em abril de 2003 (www.cnpq. br). As informações sobre os gastos do governo estadual se referem ao ano 2000 e foram obtidas na página do Ministério da Ciência e Tecnologia, MCT/seção indicadores de CeT, acessada em julho de 2002 (www.mct.gov.br). Para o número de habitantes, em 2000, utilizaram-se dados do IBGE, disponibilizados na página do $\mathrm{CNPq} /$ Resenha Estatística 1995-2000, acessada em abril de 2003 (www.cnpq.br).

- Percentual de Gasto em CeT: percentual da receita orçamentária do estado aplicada em ciência e tecnologia. Os dados se referem ao percentual médio dos últimos três anos para os quais se têm informações disponíveis (1995-1997) e foram obtidos na página do $\mathrm{MCT} /$ seção Indicadores de CeT, acessada em julho de 2002.

\section{B) Produção científicA E teCNOlÓGicA}

O sucesso inovador de países, regiões e empresas, que se traduz na modificação e melhoria incremental de produtos e processos, está associado à capacidade criativa de seu corpo de pesquisadores, para o que contribui o nível e a qualidade da produção científica e tecnológica. Na falta de indicadores perfeitos, estes aspectos são representados, nesta pesquisa, por:

- Artigos: percentual de artigos publicados por residentes do estado e indexados pelo Institute for Scientific Information (ISI) em relação ao total de artigos brasileiros indexados pelo organismo. Dado relativo a 1999 e extraído de Albuquerque (2001).

- Patentes: percentual das patentes de residentes do estado depositadas no Instituto Nacional da Propriedade Intelectual (Inpi) em relação ao total de patentes de brasileiros. Dado relativo a 1996 (dado disponível mais recente) e extraído de Albuquerque (2001).

\section{C) BASE educacional E disponibilidade DE} RECURSOS HUMANOS QUALIFICADOS

A existência de uma massa crítica que possua os conhecimentos e as habilidades cognitivas necessárias à manutenção do fluxo de inovações é condição indispensável ao sucesso inovador de um país e/ou região. O desenvolvimento socioeconômico tende a se basear, cada vez mais, na mobilização do capital de conhecimentos científicos e técnicos e nas habilidades cognitivas, as quais estão associadas, fundamentalmente, ao nível geral de educação da sociedade e à disponibilidade de profissionais com formação compatível com as exigências do desenvolvimento tecnológico.

Três indicadores foram utilizados para refletir essas habilidades.

- Taxa de escolarização de jovens: nível de escolarização da população entre 15 e 17 anos de idade, em 1999, conforme Censo 2000 do IBGE.

- Pesquisadores por milhão de habitantes: número de pesquisadores por grupo de milhão de habitantes do estado, no ano 2000. Os dados sobre o número de pesquisadores foram obtidos no Diretório dos Grupos de Pesquisa CNPqVersão 2000, disponibilizada na página do órgão e acessada em abril de 2003*.

- Pessoal de nível superior por empresa: número médio de funcionários das empresas com cem ou mais empregados,

\footnotetext{
"É preciso cautela para a realização da pesquisa direta no Diretório dos Grupos de Pesquisa do CNPq, fonte dos dados sobre o número de pesquisadores, uma vez que a unidade de pesquisa do órgão é o grupo de pesquisa e não-pesquisador, o que pode conduzir a dupla contagem de pesquisadores que participam de mais de um grupo de pesquisa. De forma a evitar esta possibilidade, considerou-se o número de pesquisadores para cada unidade da federação conforme apurado na súmula estatística do órgão.
} 


\section{Elisa Maria Pinto Rocha / Marta Araújo Tavares Ferreira}

que possuem diploma de graduação, conforme Pesquisa da Atividade Econômica Regional e Pesquisa da Atividade Econômica de São Paulo, realizadas pela Fundação Sistema Estadual de Análise de Dados do Estado de São Paulo (Seade). Dados disponibilizados na página do MCT/seção indicadores de CeT acessada em abril de 2002.

\section{D) Amplitude E DifusÃo dAS INOVAÇÕES EMPRESARIAIS}

O grau de participação e envolvimento das empresas na condução e financiamento das atividades de pesquisa é uma característica que distingue os sistemas nacionais de inovação. Diferentemente do que ocorre em países caracterizados por sistema nacional de inovação maduro, nas economias em desenvolvimento, o número de pesquisadores e o financiamento de projetos de pesquisa e desenvolvimento se concentram na esfera pública. Além de esses países apresentarem pequena amplitude da inovação no âmbito das empresas privadas, observa-se, também, que o impacto da pesquisa industrial realizada pelas firmas mostra-se relativamente baixo, em termos do conteúdo tecnológico dos produtos e serviços por elas comercializados.

Embora esses fatores acabem por imprimir fragilidades ao sistema de inovação das economias em desenvolvimento, observam-se, recentemente, nesses países, alguns movimentos potencialmente promissores e capazes de amenizar parte dessas vulnerabilidades. Esse é o caso das incubadoras de empresas de base tecnológica, que,em geral, surgem da reunião de esforços públicos e privados e tendem a contribuir para maior interação entre ciência e tecnologia e entre universidade e empresa.

No Brasil a sistematização de dados e informações sobre inovação tecnológica empresarial no nível dos estados da federação é ainda muito precária e limitada, o que impõe dificuldades adicionais à tarefa de representar, nos sistemas estaduais de inovação, os aspectos relacionados à dimensão abrangência e difusão da inovação tecnológica empresarial.

Para a construção do ICTeI, selecionaram-se como indicadores proxies dessa dimensão:

- Participação das empresas inovadoras no total de empresas: número de empresas inovadoras sediadas no estado sobre o número total de empresas do estado, conforme apurado pela Fundação Seade. Segundo os levantamentos da instituição, é inovadora a empresa que introduziu uma inovação tecnológica, de produto ou de processo, nos últimos cinco anos. As amostras da pesquisa da Fundação são compostas de empresas que possuem cem ou mais empregados; portanto, representam apenas uma parcela do universo de empresas de cada estado. Os dados correspondem à data de realização dos levantamentos (1999 para Rio de Janeiro, Espírito Santo e Santa Catarina, 1998 para Minas Gerais e Rio Grande do Sul e 1996 para São Paulo) e foram extraídos da página do $\mathrm{MCT} /$ seção indicadores de CeT, acessada em abril de 2002.

- Incubadoras de empresas: número de incubadoras de empresas existentes no estado sobre o total do país, em porcentual, conforme levantamento da Associação Nacional de Entidades Promotoras de Empreendimentos de Tecnologias Avançadas (Anprotec), em 2001. Dados disponibilizados na página da associação/seção Panorama 200, acessada em julho de 2002 (www. anprotec.org.br).

- Exportação de produtos intensivos em tecnologia: percentual das vendas externas de produtos tecnologicamente mais sofisticados em relação ao total da exportação do estado, em 2002 (a partir de tipologia utilizada pelo Iedi). Os dados foram extraídos da base de dados do Ministério do Desenvolvimento, Indústria e Comércio Exterior (MDIC/Sistema Alice), acessada em março de 2003 (www.aliceweb.desenvolvimento.gov.br).

Os valores observados para os indicadores representativos das dimensões do ICTeI encontram-se dispostos na tabela 1 . A partir deles, são calculados os índices indicadores relativos a cada um dos 10 indicadores; após, são calculados os índices sintéticos representativos de cada uma das quatro dimensões; por último, calcula-se o índice geral (ICTeI), conforme mostrado a seguir.

a) Índice indicador - obtido a partir da fórmula de cálculo do Technology Achievement Index, TAI-PNUD, adaptado para os indicadores que representam as dimensões do sistema de inovação estadual:

$$
\mathrm{II} i j=\mathrm{X} i j-\mathrm{X} i j \mathrm{mi} /(\mathrm{X} i j \mathrm{ma}-\mathrm{X} i j)
$$

em que: $i$ se refere a cada um dos 10 indicadores; e $j$ se refere a cada um dos 7 estados; IIij é o índice indicador i para o estado j; Xij corresponde ao valor observado do indicador $i$ para o estado $j$; Xijmi se refere ao valor mínimo observado do indicador $i$ para o estado $j$; Xijma corresponde ao valor máximo observado do indicador $i$ para o estado $j$.

Os valores assim obtidos variam entre "zero" (0) e "um" (1), sendo que o "um" corresponde à melhor situação relativa do estado para aquele indicador específico e "zero" corresponde à pior situação relativa. Ou seja, os índices indicadores servem de parâmetro para a identificação da 
posição relativa de cada estado em relação aos outros estados, no que diz respeito àquele indicador específico.

b) Índice sintético da dimensão - corresponde à média dos índices indicadores da dimensão para cada estado. Portanto, serve de parâmetro para a identificação da posição relativa do estado ante os demais, no que diz respeito àquela dimensão específica.

$$
\mathrm{IS} u j=\mathrm{X}(\mathrm{II} i j)
$$

onde: ISuj é o índice sintético da dimensão $u$ para o estado $j$; X (IIij) corresponde à média dos índices indicadores da dimensão $u$ para o estado $j$;

c) Índice de ciência, tecnologia e inovação (ICTeI) - é o índice geral que correspondente à média dos índices sintéticos de cada dimensão para cada estado. Possibilita a caracterização geral e a ordenação dos estados no que se refere ao sistema estadual de ciência, tecnologia e inovação. Quanto maior o valor do índice geral, mais favorável é a posição do sistema de inovação do estado em relação aos demais.

$$
\mathrm{ICTeIj}=\mathrm{M}(\mathrm{IS} u j)
$$

onde: ICTelj é índice geral de ciência, tecnologia e inovação para o estado $\mathrm{j}$ e M (ISuj) corresponde à média do índice sintético das dimensões para cada estado.

Os valores obtidos para os 10 índices indicadores, para os índices sintéticos das dimensões e para o índice geral (ICTeI), encontram-se dispostos na tabela 1 e são analisados na próxima seção.

\section{SÍNTESE DOS RESULTADOS GERAIS E PRINCIPAIS CONCLUSÕES}

Primeiramente, chama atenção o elevado nível de realização tecnológica do sistema de inovação de São Paulo, primeiro colocado no ranking geral (maior ICTeI), que detém as mais favoráveis posições em todas as dimensões - os valores dos índices sintéticos do estado ante os demais o colocam nas duas primeiras posições nas quatro dimensões que caracterizam os sistemas de inovação regionais.

Embora em menor proporção do que São Paulo, o sistema de inovação do Rio de Janeiro, Rio Grande do Sul e de Santa Catarina ocupam lugar de destaque no ranking geral. O Rio de Janeiro apresenta posição mais favorável do que São Paulo no que se refere à prioridade governamental atribuída a CeT, base educacional e disponibilidade de recursos humanos qualificados. No caso do Rio Grande do Sul, chama atenção a posição favorável do estado em termos de amplitude e difusão das inovações empresariais, em função, principalmente, do expressivo número de incubadoras de empresas de base tecnológica no estado - que responde, sozinho, por quase um terço das incubadoras de empresas do país. Já Santa Catarina, unidade da federação em que o governo estadual aplica o maior percentual relativo da receita orçamentária em CeT - o que, em certa medida, ameniza o baixo gasto per capita governamental na atividade - é o estado que apresenta a segunda maior participação relativa de empresas inovadoras no conjunto da economia estadual (tabelas 1 e 2; figura 1).

A posição do Paraná e de Minas Gerais apresenta-se menos confortável, e no caso específico de Minas Gerais, a terceira economia do país em termos de capacidade econômica de produção, ocupa a penúltima posição no plano da ciência, tecnologia e inovação (tabela 2; figura 1).

A comparação dos índices indicadores e dos índices sintéticos de Minas Gerais com os demais estados aponta a ocorrência de fragilidades que estão a merecer maior atenção por parte dos formuladores da política de ciência e tecnologia: baixa prioridade governamental a CeT, pequena participação de empresas inovadoras na economia estadual e baixa disponibilidade de pesquisadores (tabela 2).

No que se refere ao primeiro aspecto, observa-se que, dentre os estados do Sudeste-Sul, Minas Gerais é o que apresenta o mais baixo índice sintético no que se refere à prioridade governamental em CeT. $\mathrm{O}$ gasto per capita governamental - que inclui o dispêndio do tesouro estadual com a função CeT e as transferências de recursos federais via CNPq - é de apenas $\mathrm{R} \$ 5,44$ por habitante. Tão preocupante quanto esta é a observação de que o gasto do governo mineiro em CeT não chega a representar sequer $1 \%$ da receita orçamentária do estado (tabelas 1 e 2).

No que diz respeito à participação de empresas inovadoras no conjunto de empresas do estado, Minas Gerais apresenta a pior posição relativa dentre os estados da região SudesteSul - apenas 31\% de grandes e médias empresas sediadas no estado introduziram no mercado produtos e/ou processos tecnologicamente novos (tabelas 1 e 2).

Quanto ao terceiro ponto, vale observar que, embora o contingente de pesquisadores mineiros seja o terceiro maior do país (4.368 pesquisadores no estado e 50.690 no país, em 2000), Minas Gerais dispõe de apenas 244,48 pesquisadores por milhão de habitantes, número bastante modesto em relação aos demais estados do Sudeste-Sul do país. 


\section{Elisa Maria Pinto Rocha / Marta Araújo Tavares Ferreira}

\section{TABELA 1}

Valor observado: indicadores componentes do índice de ciência, tecnologia e inovação para os estados das regiões Sul e Sudeste do Brasil

\begin{tabular}{|c|c|c|c|c|c|c|c|c|c|c|}
\hline \multirow{3}{*}{ Estados } & \multicolumn{10}{|c|}{ Dimensões do Índice de Ciência, Tecnologia e Inovação (ICTel) } \\
\hline & \multicolumn{2}{|c|}{$\begin{array}{c}\text { Prioridade } \\
\text { Governamental em } \\
\text { Ciência e Tecnologia (1) }\end{array}$} & \multicolumn{2}{|c|}{$\begin{array}{l}\text { Produção Científica } \\
\text { e Tecnológica (2) }\end{array}$} & \multicolumn{3}{|c|}{$\begin{array}{c}\text { Base Educacional e } \\
\begin{array}{c}\text { Disponibilidade de Recursos } \\
\text { Humanos Qualificados (3) }\end{array}\end{array}$} & \multicolumn{3}{|c|}{ Amplitude e Difusão de Inovações (4) } \\
\hline & $\begin{array}{l}\text { Gasto P.Capita } \\
\text { Governamental }\end{array}$ & $\begin{array}{c}\text { Gasto CeT } \\
\text { sobre Receita } \\
\text { Total (\%) }\end{array}$ & $\begin{array}{c}\text { Artigos } \\
\text { Indexados pelo } \\
\text { ISI (Part \%) }\end{array}$ & $\begin{array}{c}\text { Patentes } \\
\text { Depositadas } \\
\text { no INPI } \\
\text { (Part \%) }\end{array}$ & $\begin{array}{l}\text { Taxa de } \\
\text { Escolarização } \\
\text { de Jovens (\%) }\end{array}$ & $\begin{array}{c}N^{\circ} \text { de } \\
\text { Pesquisadores } \\
\text { (p/milhão hab) }\end{array}$ & $\begin{array}{l}\text { Pess.Niv. } \\
\text { Superior por } \\
\text { Empresa }\left(n^{\circ}\right)\end{array}$ & $\begin{array}{l}\text { Part.Empresas } \\
\text { Inovadoras (\%) }\end{array}$ & $\begin{array}{c}\text { Part. } \\
\text { Incubadoras } \\
\text { de Empresas } \\
\text { (\% total BR) }\end{array}$ & $\begin{array}{c}\text { Exportações } \\
\text { Prods. } \\
\text { Intensivos } \\
\text { Tecnol. (\%) }\end{array}$ \\
\hline São Paulo & 17,92 & 1,03 & 48,65 & 56,09 & 83,90 & 413,83 & 4,20 & 55,20 & 24,00 & 48,33 \\
\hline Rio de Janeiro & 17,56 & 1,47 & 20,02 & 6,63 & 6,63 & 511,45 & 3,90 & 31,10 & 31,10 & 7,24 \\
\hline Minas Gerais & 7,24 & 0,80 & 11,51 & 8,42 & 75,10 & 244,48 & 2,70 & 31,00 & 12,67 & 10,39 \\
\hline Rio G. do Sul & 11,35 & 1,09 & 7,72 & 11,50 & 76,20 & 487,64 & 2,90 & 46,60 & 28,67 & 14,70 \\
\hline Paraná & 9,27 & 0,58 & 5,60 & 5,60 & 74,50 & 357,28 & 2,80 & 32,10 & 5,33 & 30,86 \\
\hline Santa Catarina & 3,80 & 3,17 & 3,31 & 7,34 & 74,60 & 393,49 & 2,80 & 54,40 & 6,00 & 26,47 \\
\hline Espírito Santo & 5,48 & 1,03 & 0,59 & 1,14 & 73,10 & 141,55 & 2,50 & 32,00 & 0,67 & 0,09 \\
\hline
\end{tabular}

Fonte: Albuquerque, Eduardo da M.(2000); Censo 2000 - IBGE; Indicadores de CeT do

Ministério da Ciência e Tecnologia e Anprotec (elaboração da autora).

(1) Gasto Per Capita Governamental em CeT em 2000, segundo Indicadores de Ciência e Tecnologia/MCT, expresso em R\$1,00 de 1999 (dados preliminares); trata-se do gasto federal do CNPq em apoio e fomento à pesquisa mais o gasto do governo estadual em ciência e tecnologia. Gasto/ Receita Total se refere ao valor do dispêndio estadual em CeT como percentual da receita total do estado, conforme Indicadores de Ciência e Tecnologia/MCT, relativo à média do período 1995-1997.

(2) Artigos: participação do estado no total do país, em 1999, conforme Albuquerque (2001); trata-se de artigos de autores do estado indexados pelo ISI, no total de artigos de autores brasileiros indexados pelo Instituto nas áreas de ciências biológicas e da saúde, ciências agrárias, engenharias e ciência da computação, ciências exatas e da terra. Patentes: particpação do estado no total do país, em 1996, conforme Albuquerque (2001); tratase das patentes depositadas no Inpi.

(3) Taxa de Escolarização de Jovens corresponde à população entre 15-19 anos, em 1999, conforme Censo 2000 do IBGE (tabulação avançada). Número de Pesquisadores por grupo de milhão de habitantes em 2000, segundo Diretório dos Grupos de Pesquisa no Brasil/CNPq-2000; e Censo do IBGE -2000, para população residente nas unidades da federação. Pessoal de Nível Superior por empresa, segundo Indicadores de Ciência e Tecnologia do MCT; dados obtidos a partir da Pesquisa Paer/Fundação Seade, cujos levantamentos foram realizados em 1999 no caso dos estados do Espírito Santo, Rio de Janeiro e Santa Catarina; em 1998 nos estados de Minas Gerais e Rio Grande do Sul; e em 1996 no caso de São Paulo.

(4) Participação das Empresas Inovadoras no total das empresas do estado, segundo Indicadores de Ciência e Tecnologia/MCT; dados obtidos a partir da pesquisa Paer/Fundação Seade, cujos levantamentos foram realizados em 1999 nos estados do casos do Espírito Santo, Rio de Janeiro e Santa Catarina; em 1998 nos estados de Minas Gerais e Rio Grande do Sul; e em 1996 no estado de São Paulo. Incubadoras de Empresas corresponde ao percentual do número de incubadoras de empresas do estado sobre o número total de incubadoras de empresas do país, segundo levantamento da Anprotec, em 2001. Participação das Exportações de Produtos Intensivos em Tecnologia, corresponde ao valor das vendas externas dos capítulos 30 (produtos farmacêutivos), 48 (papel e celulose), 84 (máquinas e aparelhos mecânicos), 85 (máquinas, aparelhos elétricos e de telecomunicação), 86 89 (veículos automóveis, tratores, material de transporte, aeronaves e aparelhos aeroespaciais), e 90-91 (instrumentos ópticos e de precisão), sobre o total exportado pelo estado, em 2002. 
FIGURA 1

Posição relativa dos estados do Sudeste-Sul do país no plano da ciência, tecnologia e inovação (ordem decrescente do valor do ICTeI)

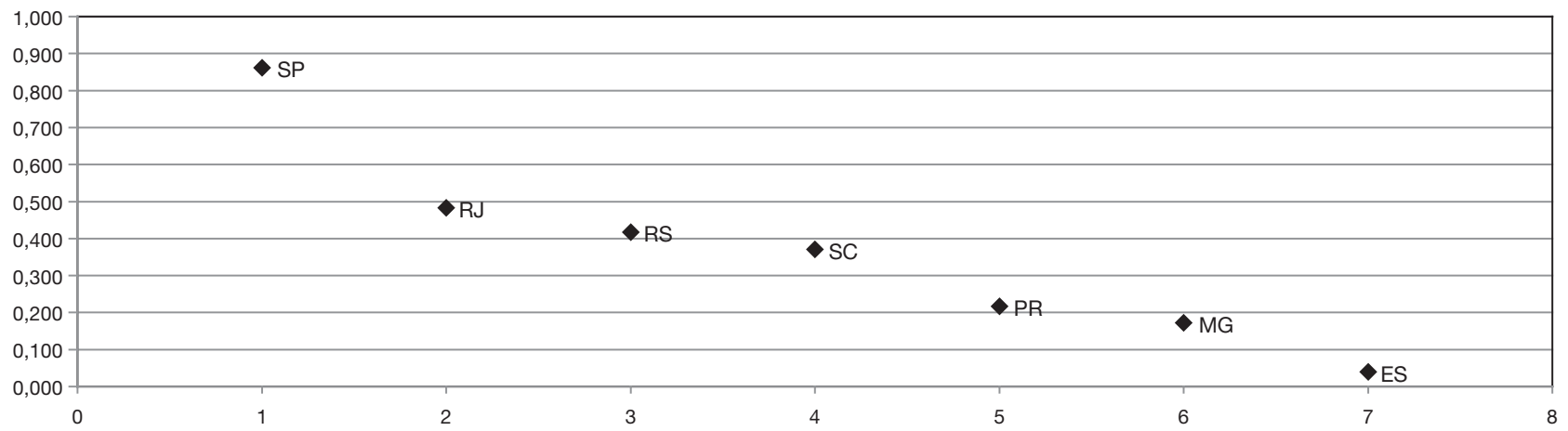

Fonte: elaboração da autora.

TABELA 2

Índice indicador, índice sintético e índice de ciência, tecnologia e inovação dos estados das regiões Sul e Sudeste do Brasil

\begin{tabular}{|c|c|c|c|c|c|c|c|c|c|c|c|c|c|c|c|c|}
\hline \multirow[b]{3}{*}{ Estados } & \multicolumn{3}{|c|}{$\begin{array}{c}\text { Prioridade } \\
\text { Governamental em } \\
\text { Ciência e Tecnologia (1) }\end{array}$} & \multicolumn{3}{|c|}{$\begin{array}{l}\text { Produção Científica } \\
\text { e Tecnológica (2) }\end{array}$} & \multicolumn{4}{|c|}{$\begin{array}{c}\text { Base Educacional e } \\
\text { Disponibilidade de Recursos } \\
\text { Humanos Qualificados (3) }\end{array}$} & \multicolumn{4}{|c|}{$\begin{array}{l}\text { Amplitude e Difusão } \\
\text { de Inovações (4) }\end{array}$} & \multirow{2}{*}{\multicolumn{2}{|c|}{$\begin{array}{c}\text { Índice de Ciência, } \\
\text { Tecnologia e } \\
\text { Inovação } \\
\text { ICTel }\end{array}$}} \\
\hline & \multicolumn{2}{|c|}{ Índice Indicador } & \multirow[b]{2}{*}{$\begin{array}{l}\text { Índice } \\
\text { Sintético }\end{array}$} & \multicolumn{2}{|c|}{ Índice Indicador } & \multirow[b]{2}{*}{$\begin{array}{l}\text { Índice } \\
\text { Sintético }\end{array}$} & \multicolumn{3}{|c|}{ Índice Indicador } & \multirow[b]{2}{*}{$\begin{array}{l}\text { Índice } \\
\text { Sintético }\end{array}$} & \multicolumn{3}{|c|}{ Índice Indicador } & \multirow[b]{2}{*}{$\begin{array}{l}\text { Índice } \\
\text { Sintético }\end{array}$} & & \\
\hline & $\begin{array}{l}\text { Gasto } \\
\text { P.Capita } \\
\text { Gover- } \\
\text { namental }\end{array}$ & $\begin{array}{c}\text { Gasto } \\
\text { CeT sobre } \\
\text { Receita } \\
\text { Total (\%) }\end{array}$ & & $\begin{array}{c}\text { Artigos } \\
\text { Indexados } \\
\text { pelo ISI } \\
\text { (Part \%) }\end{array}$ & $\begin{array}{l}\text { Patentes } \\
\text { Depo- } \\
\text { sitadas } \\
\text { no INPI } \\
\text { (Part \%) }\end{array}$ & & $\begin{array}{l}\text { Taxa de } \\
\text { Escolari- } \\
\text { zação de } \\
\text { Jovens (\%) }\end{array}$ & $\begin{array}{l}N^{0} \text { de } \\
\text { Pesqui- } \\
\text { sadores } \\
\text { (p/mi- } \\
\text { Ihão hab) }\end{array}$ & $\begin{array}{l}\text { Pess.Niv. } \\
\text { Superior } \\
\text { por Em- } \\
\text { presa }\left(n^{\circ}\right)\end{array}$ & & $\begin{array}{l}\text { Part.Em- } \\
\text { presas } \\
\text { Inovado- } \\
\text { ras (\%) }\end{array}$ & $\begin{array}{c}\text { Part. Incu- } \\
\text { badoras } \\
\text { de Em- } \\
\text { presas (\% } \\
\text { total BR) }\end{array}$ & $\begin{array}{l}\text { Expor- } \\
\text { tações } \\
\text { Prods. } \\
\text { Intensi- } \\
\text { vos Tec- } \\
\text { nol. (\%) }\end{array}$ & & Valor & Posição \\
\hline $\begin{array}{l}\text { São } \\
\text { Paulo }\end{array}$ & 1,000 & 0,176 & 0,588 & 1,000 & 1,000 & 1,000 & 1,000 & 0,736 & 1,000 & 0,912 & 1,000 & 0,833 & 1,000 & 0,944 & 0,861 & $1^{\circ}$ \\
\hline $\begin{array}{l}\text { Rio de } \\
\text { Janeiro }\end{array}$ & 0,974 & 0,344 & 0,659 & 0,404 & 0,100 & 0,252 & 0,926 & 1,000 & 0,824 & 0,916 & 0,004 & 0,167 & 0,148 & 0,106 & 0,484 & $2^{\circ}$ \\
\hline Paraná & 0,387 & 0,000 & 0,194 & 0,104 & 0,081 & 0,093 & 0,130 & 0,583 & 0,176 & 0,296 & 0,045 & 0,167 & 0,638 & 0,283 & 0,217 & $5^{\circ}$ \\
\hline $\begin{array}{l}\text { Santa } \\
\text { Catarina }\end{array}$ & 0,000 & 1,000 & 0,500 & 0,057 & 0,113 & 0,085 & 0,139 & 0,681 & 0,176 & 0,332 & 0,967 & 0,190 & 0,547 & 0,568 & 0,371 & $6^{\circ}$ \\
\hline $\begin{array}{l}\text { Espírito } \\
\text { Santo }\end{array}$ & 0,119 & 0,175 & 0,147 & 0,000 & 0,000 & 0,000 & 0,000 & 0,000 & 0,000 & 0,000 & 0,041 & 0,000 & 0,000 & 0,014 & 0,040 & $7^{\circ}$ \\
\hline
\end{tabular}

Fonte: elaboração da autora, a partir de dados básicos de Albuquerque (2001); CNPq, IBGE, MCT, MDIC, Anprotec.

Observação: considerar as notas de rodapé da tabela 1 como válidas também para a tabela 2. 


\section{Elisa Maria Pinto Rocha / Marta Araújo Tavares Ferreira}

Em contrapartida, é importante ressaltar que, apesar dessas fragilidades, o sistema de inovação de Minas Gerais detém posição relativamente favorável no que se refere à produção científica e tecnológica e ao potencial representado pelas incubadoras de empresas. Quando comparado aos índices indicadores dos outros estados, observa-se que Minas Gerais ocupa a terceira melhor colocação no caso desses três indicadores. $\mathrm{O}$ estado responde por 11,5\% dos artigos brasileiros indexados pelo ISI, por 8,4\% dos depósitos de patentes no Inpi, e abriga $12,7 \%$ do total de incubadoras do país. Sem dúvida, essas oportunidades poderão se constituir credenciais relevantes para o desenvolvimento de um efetivo sistema de inovação em Minas Gerais.

Finalmente, as faixas de valores dos ICTeI sugerem a existência de pelo menos quatro "estágios" de desenvolvimento dos sistemas de inovação entre os estados do Sudeste-Sul do país. No "estágio" mais avançado (ICTeI acima de 0,8) estaria posicionado São Paulo. Rio de Janeiro, Rio Grande do Sul e Santa Catarina (ICTeI entre 0,4 e 0,8) ocupariam posições imediatamente abaixo de São Paulo. Paraná e Minas Gerais ocupariam posições menos confortáveis (ICTeI entre 0,1 e 0,4), enquanto o Espírito Santo corresponderia a um estágio menos avançado (ICTeI abaixo de 0,1) (tabela 2; figura 1).

Artigo recebido em 15-09-2004 e aceito para publicação em 29-03-2005.

\section{REFERÊNCIAS}

AGUiAR, Afrânio; GONÇALVES, Carlos Alberto. Política de desenvolvimento científico e tecnológico: qual a realidade de Minas Gerais? Revista Perspectivas em Ciência da Informação, Belo Horizonte, v. 5, n. 2, p. 151-166, jul./dez. 2000.

ALBUQUERQUE, Eduardo M. Sistema estadual de inovação de Minas Gerais: um balanço introdutório e uma discussão do papel (real e potencial) da FAPEMIG para a sua construção. Versão preliminar. Belo Horizonte : [s. n.], 2001. 105 p.
CENDÓN, Beatriz V. Bases de dados de informação para negócios. Ciência da Informação, Brasília, v. 31, n. 2, p. 30-43, maio/ago. 2002.

DINIZ, C. C., LEMOS, M. B. Sistemas locais de inovação: o caso de Minas Gerais. In: CASSIOLATO, José Eduardo LASTRES, Helena M. M. (Org.). Globalização e inovação globalizada: experiências de sistemas locais no Mercosul. Brasília : IBICT, 1999.

FREEMAN, C.; SOETE, L. The economics of industrial innovation. London : Pinter, 1997.

LASTRES, Helena M. M. ALBAGLI, Sarita (Org.). Informação e globalização na era do conhecimento. Rio de Janeiro : Campus, 1999. 318 p.

MACIAS-CHAPULA, César A. O papel da informetria e da cienciometria e sua perspectiva nacional e internacional. Ciência da Informação, Brasília, v. 27, n.2, p. 134-140, maio/ago. 1998.

MONTALli, Kátia M. L.; CAMPELO, Bernadete dos S. Fontes de informação sobre companhias e produtos industriais: uma revisão de literatura. Ciência da Informação, Brasília, v. 26, n. 3, p. 321-326, set./ dez. 1997.

NELSON, R. (Ed.). National innovation systems: a comparative analysis. New York : Oxford University, 1993.

SOUZA, Terezinha de F. C.; BORGES, Mônica E. N. Instituições provedoras de informação tecnológica no Brasil: análise do potencial para atuação com informação para negócios. Ciência da Informação, Brasília, v. 25, n. 1, p. 52-58, jan./abr. 1996.

SPINAK, Ernesto. Indicadores cienciométricos. Ciência da Informação, Brasília, v. 27, n. 2, p. 141-148, maio/ago. 1998.

ROUSSEAU, Ronald. Indicadores bibliométricos e econométricos para a avaliação de instituições científicas. Ciência da Informação, Brasília, v. 27, n. 2, p. 149-158, maio/ago. 1998.

UNITED NATIONS. Development programme: human development report. New York : Oxford University, 2001.

VANTI, Nadia A. P. Da bibliometria à webometria: uma exploração conceitual dos mecanismos utilizados para medir o registro da informação e a difusão do conhecimento. Ciência da Informação, Brasília, v. 31, n. 2, p. 152-162, maio/ago. 2002.

WORMEll, Irene. Informetria: explorando bases de dados como instrumentos de análise. Ciência da Informaç̧ão, Brasília, v. 27, n. 2, p. 210-216, maio/ago. 1998. 